\title{
Hallazgo casual de origen anómalo de la arteria coronaria derecha desde la arteria pulmonar en un lactante
} An incidentally detected anomalous origin of the right coronary artery from the
pulmonary artery in an infant

\author{
Dra. Sezen Ugan Atik, Prof. Dr. Levent Saltik, Prof. Asoc. Dr. Kazım Oztarhan ${ }^{b}$ y Dra. Helen Bornaun
}

\begin{abstract}
RESUMEN
Elorigen anómalo aislado de la arteria coronaria derecha desde la arteria pulmonar principal es una anomalía congénita rara y se han notificado pocos casos en la población pediátrica. En este artículo informamos el caso asintomático de un lactante varón de dos meses de edad al que se le diagnosticó origen anómalo de la arteria coronaria derecha desde la arteria pulmonar principal durante la evaluación realizada para detectar anomalías cardíacas. Debido a la sospecha durante una ecocardiografía, se realizaron un cateterismo cardíaco y una angiografía coronaria para verificar el diagnóstico del origen anómalo de la arteria coronaria derecha desde la arteria pulmonar principal. El paciente se sometió a la cirugía y estaba en buen estado en el seguimiento a los dos meses. El diagnóstico temprano podría evitar que los pacientes tengan complicaciones cardiovasculares.

Palabras clave: Ecocardiografía, arteria coronaria, arteriapulmonar, anomalías, cateterismo cardíaco.
\end{abstract}

http: / / dx.doi.org/10.5546/ aap.2018.e102

Texto completo en inglés:

http: / / dx.doi.org/10.5546/ aap.2018.eng.e102

Cómo citar: Ugan Atik S, Saltik L, Oztarhan K, et al. Hallazgo casual de origen anómalo de la arteria coronaria derecha desde la arteria pulmonar en un lactante. Arch Argent Pediatr 2018;116(1):e102-e105.

\section{INTRODUCCIÓN}

El origen anómalo aislado de la arteria coronaria derecha desde la arteria pulmonar principal (ARCAPA, por sus siglas en inglés) es una anomalía congénita rara y se han notificado pocos casos en la población pediátrica. ${ }^{1}$ La primera serie de pacientes fue descrita por Brooks en

a. Departamento de Cardiología Pediátrica, Facultad de Medicina de Cerrahpasa, İstanbul Üniversitesi, Estambul, Turquía.

b. Hospital de Formación e Investigación Kanunî Sultan Süleyman, Estambul, Turquía.

\section{Correspondencia:}

Dra. Sezen Ugan Atik: sezenugan@hotmail.com

Financiamiento: Ninguno.

Conflicto de intereses: Ninguno que declarar.

Recibido: 25-3-2017

Aceptado: 6-9-2017
1885. ${ }^{2}$ Según una revisión bibliográfica realizada por Modi y col. en 2010, se diagnosticaron 20 lactantes ( $\leq 1$ año de edad), 44 en niños ( $\leq 18$ años de edad), 17 adultos ( $>60$ años de edad) y 8 en los cuales no se registró la edad. ${ }^{3}$ Los pacientes con ARCAPA suelen ser asintomáticos, aunque se han notificado algunos casos de muerte súbita, miocardiopatía, angina de pecho y síncope. ${ }^{4}$ En este artículo informamos el caso asintomático de un lactante varón de dos meses de edad al que se le diagnosticó ARCAPA durante la evaluación realizada para detectar anomalías cardíacas.

\section{CASO}

Derivaron a nuestros consultorios externos a un lactante varón de dos meses de edad $(4,5 \mathrm{~kg})$ para una evaluación cardíaca. El bebé nació a las 38 semanas de gestación por cesárea con un peso de 3200 g y recibió el alta dentro de las 24 h tras un chequeo de rutina. Durante la hospitalización inicial, la madre indicó preocupación debido a que el bebé tenía cianosis perioral pero la saturación era normal. En el examen físico, se observó soplo sistólico 1 / 6 inespecífico en el costado izquierdo del esternón. No se detectaron otras anomalías asociadas durante el examen físico. Sus análisis de sangre estaban dentro de los límites normales. En la radiografía de tórax se observó cardiomegalia leve. El electrocardiograma mostró taquicardia sinusal con desviación del eje hacia la derecha. En la ecocardiografía, las funciones ventriculares izquierda y derecha eran normales; se detectaron señales múltiples en la ecografía Doppler color dentro del tabique interventricular sumamente sospechosas de arterias colaterales intracoronarias (Figura 1a). La arteria coronaria izquierda estaba levemente dilatada y el orificio de la arteria coronaria derecha ingresaba a la arteria pulmonar unos pocos milímetros a nivel distal de la válvula pulmonar (Figura 1b). Se detectó flujo fistuloso desde el orificio de la arteria coronaria derecha hacia la arteria pulmonar mediante la ecografía Doppler color. 
Se realizaron un cateterismo cardíaco y una angiografía coronaria para verificar el diagnóstico de ARCAPA. La angiografía selectiva de la arteria coronaria izquierda mostró llenado retrógrado de la arteria coronaria derecha a través de las arterias colaterales desde la arteria coronaria izquierda y el orificio de la arteria coronaria derecha conectado a la arteria pulmonar (Figura 2). Con esta angiografía se confirmó el diagnóstico de ARCAPA. Tras analizar el caso y ante el riesgo potencial conocido de muerte súbita, se decidió enviar al paciente para que le realizaran una cirugía de reimplante directo de la arteria coronaria derecha. Después del reimplante de la arteria coronaria derecha en la aorta, que se realizó en otra institución, el paciente se encontraba bien durante los siete meses de seguimiento.

\section{DISCUSIÓN}

Las anomalías congénitas de las arterias coronarias son malformaciones raras en la población general. Según Greenberg y col., las anomalías más importantes que llevan a perfusión miocárdica anormal son el origen anómalo desde la arteria pulmonar, el origen de una arteria coronaria desde el seno contrario o no coronario, el puente miocárdico y la fístula

FIGURA 1a: Señales múltiples de la ecografía Doppler color dentro del tabique interventricular

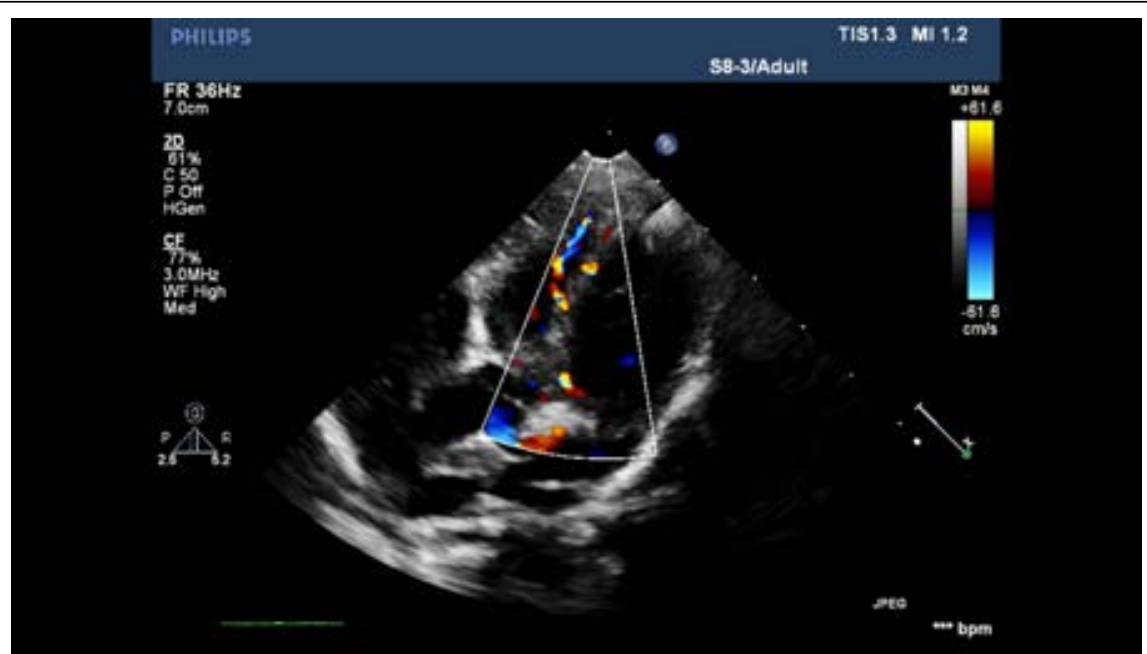

FIGURA 1b: Flujo fistuloso (flechas) desde el orificio de la arteria coronaria derecha hacia la arteria pulmonar detectado mediante ecografía Doppler color

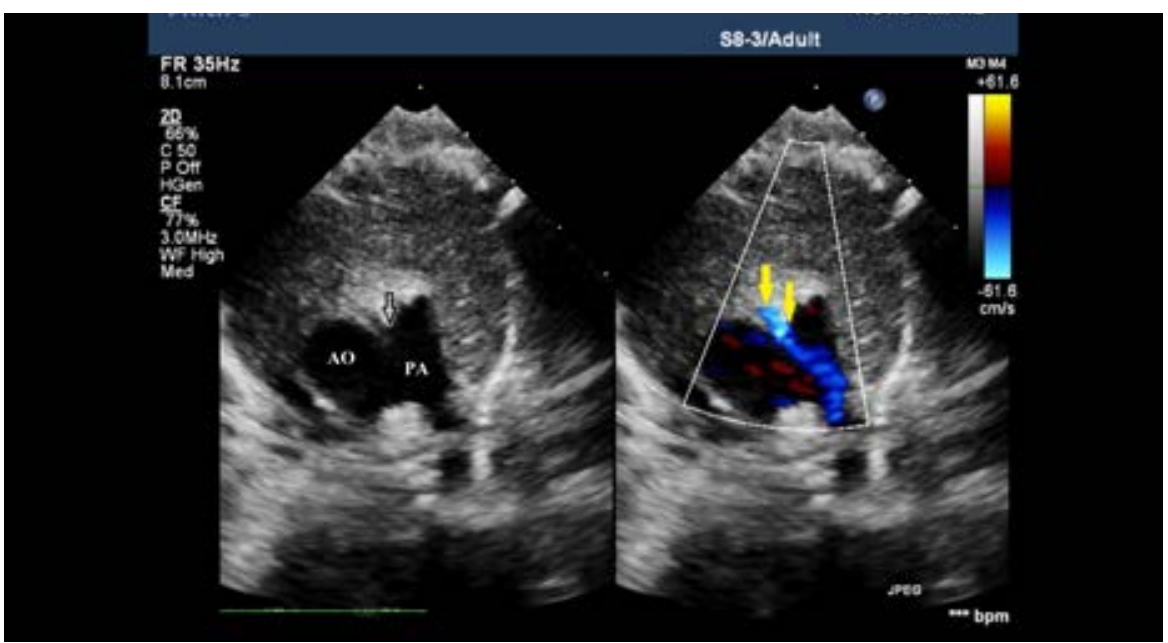


de la arteria coronaria. Entre los casos de origen anómalo desde la arteria pulmonar, el origen anómalo de la arteria coronaria izquierda desde la arteria pulmonar (ALCAPA, por sus siglas en inglés) es más frecuente. ${ }^{5}$ Los casos de ARCAPA son más raros que los de ALCAPA (se han notificado solo 200 casos en total). ${ }^{1}$ Dado que muchos pacientes no presentan síntomas, la incidencia real de esta anomalía coronaria podría ser mayor. ${ }^{2}$ En el $70 \%$ de los casos notificados, esta fue una anomalía aislada. Sin embargo, anteriormente se han notificado defectos cardíacos congénitos concomitantes, tales como ventana aortopulmonar y tetralogía de Fallot, en asociación con ARCAPA. ${ }^{6,7}$

En general, estos pacientes no tienen síntomas hasta la adultez debido a la amplia colateralización entre ambos sistemas coronarios, la cual conserva una adecuada función ventricular. ${ }^{8}$ La presentación de ARCAPA no es uniforme; los pacientes podrían tener hallazgos inespecíficos en el examen físico o disnea, fatiga, insuficiencia cardíaca congestiva, angina de pecho, infarto de miocardio e incluso paro cardíaco súbito como un primer síntoma. ${ }^{8}$ Es posible que la evaluación cardíaca se realice como resultado de un síntoma inespecífico o debido a hallazgos durante el examen físico, como un soplo. En el caso de nuestro paciente, su madre indicó preocupación porque tenía cianosis perioral pero la saturación del paciente era normal y el único hallazgo físico fue un soplo sistólico 1/ 6 inespecífico en el costado izquierdo del esternón. Por otro lado, según lo informado por Lin y col., la primera presentación en un lactante podría ser insuficiencia cardíaca aguda. ${ }^{9}$

En 1985, se diagnosticó el primer caso de ARCAPA por ecocardiografía. ${ }^{10}$ Se han notificado ciertos hallazgos ecocardiográficos, como las arterias colaterales intracoronarias dentro del tabique ventricular visualizadas mediante ecografía Doppler color, como claras señales de ARCAPA. ${ }^{11}$ Además, como en el caso de nuestro paciente, las arterias coronarias dilatadas y el flujo retrógrado desde la arteria coronaria derecha hacia la arteria pulmonar

FIGURA 2: Llenado retrógrado de la arteria coronaria derecha (flechas) a través de las arterias colaterales desde la arteria coronaria izquierda y orificio de la arteria coronaria derecha conectado a la arteria pulmonar

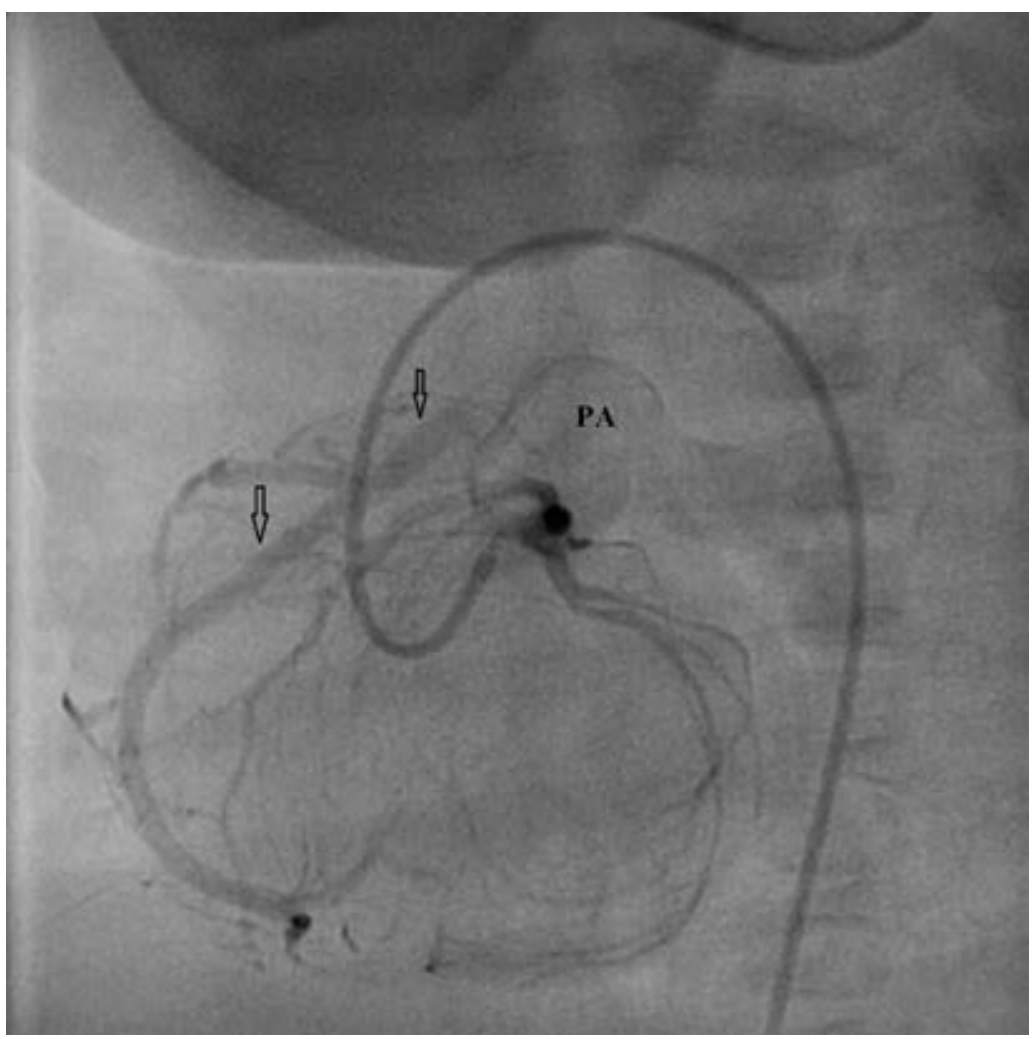


son otras manifestaciones ecocardiográficas típicas de esta afección. ${ }^{12,13}$ Podría resultar difícil realizar estudios de diagnóstico por imágenes de las arterias coronarias en algunos pacientes debido a las ventanas acústicas deficientes. La tomografía computarizada multicorte y la resonancia magnética cardíaca son modalidades de diagnóstico por imágenes no invasivas con una elevada precisión diagnóstica para detectar anomalías anatómicas en las arterias y podrían ser otras opciones para el diagnóstico. ${ }^{14}$

Las recomendaciones terapéuticas para los pacientes con ARCAPA son controvertidas. Las técnicas quirúrgicas disponibles incluyen la ligadura simple de la arteria coronaria derecha, la ligadura de la arteria coronaria derecha con injerto de revascularización coronaria de la vena safena y el reimplante de la arteria coronaria derecha en la aorta. ${ }^{15}$ Muchos autores abogan que se debe establecer un sistema de dos arterias coronarias mediante el reimplante directo de la arteria coronaria derecha en la aorta ascendente siempre que sea posible, en especial en los niños. ${ }^{5}$ En vista de estas recomendaciones, se sugirió el reimplante de la arteria coronaria derecha en la raíz aórtica inmediatamente después del diagnóstico debido al desenlace mortal potencial.

A modo de conclusión, los médicos deben estar al tanto de esta anomalía rara para realizar el diagnóstico temprano. Cuando se detectan señales múltiples en la ecografía Doppler color en el tabique interventricular en la ecocardiografía, es necesario prestar atención a los orificios en la arteria coronaria. Si se observa una arteria coronaria izquierda dilatada en el ecocardiograma, se debe sospechar la posibilidad de ARCAPA o atresia del orificio de la arteria coronaria derecha o fístula de la arteria coronaria, que son los diagnósticos diferenciales. El diagnóstico temprano podría evitar que los pacientes tengan complicaciones cardiovasculares.

\section{REFERENCIAS}

1. Chernogrivov AE, Gornostaev AA, Chernogrivov IE, et al. Anomalous origin of the right coronary artery from the pulmonary artery: surgical re-implantation into the aorta. Multimed Man Cardiothorac Surg. 2015;2015. pii. mmv024.

2. Balakrishna P, Illovsky M, Al-Saghir YM, et al. Anomalous Origin of Right Coronary Artery Originating from the Pulmonary Trunk (ARCAPA): an Incidental Finding in a Patient Presenting with Chest Pain. Cureus 2017;9(4):e1172.

3. Modi H, Ariyachaipanich A, Dia M. Anomalous origin of right coronary artery from pulmonary artery and severe mitral regurgitation due to myxomatous mitral valve disease: a case report and literature review. J Invasive Cardiol 2010;22(4):E49-55.

4. Contreras AE, Leonardi C, Lazzarin O, et al. Anomalous origin of the right coronary artery from the pulmonary artery diagnosed as an incidental finding. Congenit Heart Dis 2013;8(2):E52-5.

5. Greenberg MA, Fish BG, Spindola-Franco H. Congenital anomalies of the coronary arteries. Classification and significance. Radiol Clin North Am 1989;27(6):1127-46.

6. Williams IA, Gersony WM, Hellenbrand WE. Anomalous right coronary artery arising from the pulmonary artery: a report of 7 cases and a review of the literature. Am Heart J 2006;152(5):1004.e9-17.

7. Greenway SC, Bradley TJ, Caldarone CA, et al. Aortopulmonary window with anomalous origin of the right coronary artery from the pulmonary artery: two cases highlighting the importance of complete preoperative echocardiographic evaluation of the coronary arteries in all conotruncal anomalies. Eur J Echocardiogr 2006;7(5):379-82.

8. Radke PW, Messmer BJ, Haager PK, et al. Anomalous origin of the right coronary artery: preoperative and postoperative hemodynamics. Ann Thorac Surg 1998;66(4):1444-9.

9. Lin SM, Wu SJ, Lin HY, et al. Anomalous Origin of the Right Coronary Artery from the Pulmonary Artery in an Infant. Pediatr Neonatol 2016;57(5):446-8.

10. Suzuki K, Yokochi K, Yoshioka F, et al. Anomalous origin of the right coronary artery from the pulmonary artery: report of a case. J Cardiogr 1985;15(1):241-8.

11. Kühn A, Kasnar-Samprec J, Schreiber C, et al. Anomalous origin of the right coronary artery from the pulmonary artery (ARCAPA). Int J Cardiol 2010;139(2):e27-8.

12. Yao CT, Wang JN, Yeh CN, et al. Isolated anomalous origin of right coronary artery from the main pulmonary artery. J Card Surg 2005;20(5):487-9.

13. Hekmat V, Rao SM, Chhabra M, et al. Anomalous origin of the right coronary artery from the main pulmonary artery: diagnosis and management. Clin Cardiol 1998;21(10):773-6.

14. Shariat M, Grosse-Wortmann L, Seed M, et al. Isolated anomalous origin of the right coronary artery from the pulmonary artery in an asymptomatic 12 -year-old girl: role of MRI in depicting the anatomy, detecting the ischemic burden, and quantifying the amount of left-to-right shunt. World J Pediatr Congenit Heart Surg 2013;4(2):201-5.

15. Kautzner J, Veselka J, Rohac J. Anomalous origin of the right coronary artery from the pulmonary trunk: is surgical reimplantation into the aorta a method of choice? Clin Cardiol 1996;19(3):257-9. 